\title{
EGFR inhibition in metastatic triple negative breast cancer: a losing target
}

\begin{abstract}
Triple negative breast cancer is the most aggressive histologic subtype with an increased risk of disease recurrence and a high potential for distant metastases. Despite the drastic progress in the management of other metastatic breast cancer subtypes, the current available treatments for the metastatic triple negative breast cancer are not so promising. Cytotoxic agents remain among the only standard of care. However, certain pathways were or are being evaluated in this setting. One of the main mechanisms for cell proliferation is regulated by the epidermal growth factor receptor (EGFR) pathway, and thus it was successfully targeted in many tumors: non small cell lung cancer, colon cancer and head and neck squamous cell carcinomas. What is the rationale from inhibiting the epidermal growth factor receptor in metastatic triple negative breast cancer? What are the results of the previous trials and will there be any place for EGFR inhibitors to control this highly aggressive breast cancer subtype? In this short review of the literature, I will try to answer if there is an indication for EGFR inhibitors in the metastatic triple negative breast cancer.
\end{abstract}

Keywords: metastatic triple negative breast cancer, epidermal growth factor receptor
Volume 6 Issue 3 - 2018

\author{
Georges El Hachem \\ Department of Medical Oncology, Erasme Hospital University \\ Medical Center and Institut Jules Bordet, Belgium
}

\begin{abstract}
Correspondence: Georges El Hachem, Department of Medical Oncology, Erasme Hospital University Medical Center and Institut Jules Bordet, Brussels, Belgium,

Email george.el.hashem@hotmail.com, georges.elhachem@ bordet.be
\end{abstract}

Received: May 15, 2018| Published: May 22, 2018
Abbreviations: HR, hormone receptors; PARP, poly-ADPribose-polymerase inhibitors; $\mathrm{mTNBC}$, metastatic triple negative breast cancer; EGFR, epidermal growth factor receptor

\section{Introduction}

Triple negative breast cancer is a histologic subtype of breast carcinomas characterized by the lack of expression of hormone receptors (HR) and human epidermal growth factor receptor 2 (HER2). Fifteen percents of the breast carcinomas are triple negative. It is the most aggressive histological subtype, affecting younger age population. It is highly associated with distant recurrences despite adequate local control, mainly in the first three years following the diagnosis. In the metastatic setting, the median overall survival is around 12 months. ${ }^{1,2}$ The only available treatments still consist of conventional cytotoxic chemotherapy and many promising results were seen with poly-ADP-ribose-polymerase inhibitors (PARP) inhibitors during this last decade. Many pathways and receptors expressed in the metastatic triple negative breast cancer (mTNBC) had been the subjects of research and clinical trials: androgen receptors, epidermal growth factor receptor (EGFR), antibody drug conjugate via targeting certain surface receptors and anti-angiogenics.

\section{What is the rationale from using EGFR inhibitors in the mTNBC?}

EGFR over expression has been reported in up to $78 \%$ of triplenegative breast cancers. ${ }^{3}$ View the lack of targeted therapies in this entity of breast cancer, the researchers tried to inhibit the EGFR pathway. High EGFR copy number was associated with poor clinical outcome in TNBC suggesting that evaluation of EGFR copy number can be useful for predicting outcomes in patients with triple-negative breast cancer and selecting patients for anti-EGFR-targeted therapy. This was evaluated in many trials published in the literature. It is unknown if EGFR is a valid target because many of the phase II studies of EGFR tyrosine-kinase inhibitor in metastatic breast cancer had at most 5\% response rate. However, a major breakthrough in EGFR-targeted therapy was reported at the European Society of Medical Oncology meeting in October 2010, ${ }^{4}$ where a randomized phase III study, the BALI trial showed an overall response rate of $20 \%$ in patients with metastatic TNBC who received the cetuximab/ cisplatin combination, compared with a response rate of $10.3 \%$ in the cisplatin-alone arm. Adding cetuximab to cisplatin doubled the progression-free survival duration from 1.5 to 3.7 months (HR $0.675, \mathrm{P}=0.032) .{ }^{4}$ Although the response rate $(\mathrm{RR})$ was doubled in the combination arm, it did not meet the pre-specified primary end point of the study (up to an RR of $32 \%$ ), thus the further usage of the combination was abandoned. Moreover, the patients included in this trial were early during their disease evolution and the result obtained with the control arm is to be considered surprisingly low.

In the same perspective, the monoclonal antibody cetuximab has shown some interesting activity in patients with TNBC when combined with single-agent carboplatin [objective response rate $(\mathrm{ORR})=18]$ and with carboplatin/irinotecan $(\mathrm{ORR}=49 \%$ in the TNBC population). ${ }^{5}$ Investigators tried to identify those patients with metastatic TNBC who may benefit from cetuximab treatment, which may be correlated with lower expression of alpha-crystalline B chain (encoded by the CRYAB gene), higher expression of phosphatase and tensin (PTEN) homologue and lack of KRAS expression in patients with basal-like breast cancer. ${ }^{6}$

Another monoclonal antibody directed against EGFR, the panitumumab, was investigated in a phase II trial in combination with carboplatin and paclitaxel in patients with metastatic HER2-negative breast cancer with ER and PR expression of $<10 \%$ (clinical trial NCT01009983). According to the clinicaltrials.gov website, the last updates were posted in February 2018. Only fourteen patients were analyzed. There were no statistical analysis provided for the antitumor activity which was assessed by the objective tumor response according 
to RECIST criteria, and there is a lack of statistical analysis provided for time to progression and survival. Thus, this trial had important limitations, such as early termination leading to small numbers of participants analyzed and technical problems with measurement leading to unreliable or uninterpretable data, again as reported on the clinical trials website (clinicaltrials.gov). Another phase II trial with panitumumab, gemcitabine and carboplatin in patients with metastatic TNBC has recently suspended recruitment. The suspension of this trial was due to funding issues and was not related to safety or efficacy results.

Small-molecule TKIs against EGFR (gefitinib, afatinib and erlotinib) were also being evaluated in mTNBC. Baselga et al. ${ }^{7}$ reported that gefitinib showed minimal single-agent activity in patients with metastatic breast cancer; this was consistent with the results of a study by Dickler et al., ${ }^{8}$ which concluded that erlotinib also showed a minimal activity as a single-agent therapy for patients with metastatic breast cancer. These studies included patients with metastatic breast cancer who were not selected for the presence of activating mutations in the EGFR gene. In other tumour types, stratifying the patient population according to EGFR activation status has improved RRs with EGFR TKIs. It remains unknown whether EGFR TKIs add any value to the therapy for TNBC. ${ }^{8,9}$ However, preclinical data support the use of erlotinib or gefitinib combined with docetaxel or carboplatin in TNBC cell lines. ${ }^{9}$ Furthermore, there was no reported benefit from using the TKIs in $\mathrm{mTNBC}$, with minimal non significant clinical activity: 1 partial response in the erlotinib trial, in association with bevacizumab and ORR of $0 \%$ in the phase II trials with gefitinib and afatinib)., 7,10

\section{Conclusion}

Despite the expression of the EGFR in mTNBC, the administration of anti-EGFR therapies didn't show any efficient results. Although it is an important pathway in other cancer subtypes with impressive survival results mainly in non small cell lung cancer, it is not the case in mTNBC. Neither the early clinical data with EGFR tyrosine-kinase inhibitor in metastatic breast cancer where the response rate was around 5\%, nor the late trials that tested the EGFR antibody and TKIs in combination with cytotoxic chemotherapy showed a statistically significant objective response. This lack of benefit can be explained by the fact that the malignant cells depend on other proto-oncogenes as well as on the activation of different pathways for growth, proliferation and invasion. Unfortunately this entity of breast cancer remains fatal and poorly controlled. Newer treatment modalities are currently evaluated with promising results, mainly regarding the antibody drug conjugates and immunotherapy, as it was presented in the San Antonio meeting symposium in December 2017, and we are waiting for further data and results that are going to be discussed in the American Society of Medical Oncology (ASCO) meeting.

\section{Acknowledgements}

None.

\section{Conflict of interest}

The author declares that there is no conflict of interest.

\section{References}

1. Kassam F, Enright K, Dent R, et al. Survival Outcomes for Patients With Metastatic Triple-Negative Breast Cancer: Implications for Clinical Practice and Trial Design. Clin Breast Cancer. 2009;9(1):29-33.

2. Khosravi-Shahi P, Cabezón-Gutiérrez L, Custodio-Cabello S. Metastatic triple negative breast cancer: Optimizing treatment options, new and emerging targeted therapies. Asia Pac J Clin Oncol. 2018;14(1):32-39.

3. Gluz O, Liedtke C, Gottschalk N, et al. Triple-negative breast cancer-current status and future directions. Ann Oncol. 2009;20(12):1913-1927.

4. Baselga J. The addition of cetuximab to cisplatin increases overall response rate (ORR) and progression-free survival (PFS) in metastatic triple-negative breast cancer (TNBC): results of a randomized phase II study (BALI-1). Italy: Milan, $35^{\text {th }}$ Congress of the European Society for Medical Oncology (ESMO); 2010.

5. Vukelja S, Oshaughnessy J, Weckstein DJ. Preliminary results of a randomized phase II study of weekly irinotecan/carboplatin with or without cetuximab in patients with metastatic breast cancer. Breast Cancer Res Treat. 2007;106:308

6. Carey LA, Oshaughnessy J, Hoadley K, et al. Potential predictive markers of benefit from cetuximab in metastatic breast cancer: an analysis of two randomized phase 2 trials. Cancer research. 2009;69(24 Suppl):9-13.

7. Baselga J, Albanell J, Ruiz A, et al. Phase II and tumor pharmacodynamic study of gefitinib in patients with advanced breast cancer. $J$ Clin Oncol. 2005;23:5323-5333.

8. Dickler MN, Rugo HS, Eberle CA, et al. A phase II trial of erlotinib in combination with bevacizumab in patients with metastatic breast cancer. Clin Cancer Res. 2008;14(23):7878-7883.

9. Corkery B, Crown J, Clynes M, et al. Epidermal growth factor receptor as a potential therapeutic target in triple-negative breast cancer. Ann Oncol. 2009;20(5):862-867.

10. Schuler M, Awada A, Harter P, et al. A phase II trial to assess efficacy and safety of afatinib in extensively pretreated patients with HER2-negative metastatic breast cancer. Breast Cancer Res Treat. 2012;134(3):1149 1159. 